\title{
Preparation of Chitosan/Poly- $\gamma$-Glutamic Acid Polyelectrolyte Multilayers on Biomedical Metals for Local Antibiotic Delivery
}

\author{
Wai-Ching Liu ${ }^{1}$, Huey-Yuan Wang ${ }^{2}$, An-Ni Wang ${ }^{1}{ }^{(\mathbb{D})}$, Chih-Hsien Tseng ${ }^{1}$, Hsuan-Liang Liu ${ }^{1}$ \\ and Ren-Jei Chung $1, *$ \\ 1 Department of Chemical Engineering and Biotechnology, National Taipei University of Technology \\ (Taipei Tech.), Taipei 10608, Taiwan; criswcliu@gmail.com (W.-C.L.); dai20020223@gmail.com (A.-N.W.); \\ q1237689@hotmail.com (C.-H.T.); f10894@ntut.edu.tw (H.-L.L.) \\ 2 Department of Stomatology, MacKay Memorial Hospital, Taipei 10449, Taiwan; wang461110@yahoo.com.tw \\ * Correspondence: rjchung@ntut.edu.tw; Tel.: +886-2-8772-8701
}

Received: 14 September 2017; Accepted: 5 October 2017; Published: 7 October 2017

\begin{abstract}
Polyelectrolyte multilayer assembly is one of the most widely applied biomaterial coatings for applications from surface modification, drug delivery, tissue engineering to biomimetic extracellular environment. In this research, we propose a simple layer-wise spin coating technique to prepare chitosan/poly- $\gamma$-glutamic acid (C/PGA) polyelectrolyte multilayers (PEMs) on two different biomedical metals, 316L stainless steel (316LSS) and titanium alloy (Ti6Al4V). The multilayer coating was fabricated using oppositely charged chitosan and poly- $\gamma$-glutamic acid to deposit a total of 10, 20, or 30 multilayered films. Afterward, tetracycline was loaded by soaking the coated metals for 12 hours. The microstructure, mechanical properties, biocompatibility and drug release rate were investigated by scanning electron microscopy, contact angle measurement, MG63 cell viability and inhibition of Escherichia coli (E. coli) growth. Lastly, MG63 cell attachment was detected by fluorescence microscopy after staining with Hoechst 33258. This coating technique can prepare a layer of 2.2-6.9 $\mu \mathrm{m} \mathrm{C/PGA} \mathrm{PEMs} \mathrm{favoring} \mathrm{cell} \mathrm{attachment} \mathrm{and} \mathrm{growth.} \mathrm{Moreover,} \mathrm{tetracycline} \mathrm{was}$ released from C/PGA PEMs and inhibited the growth of E. coli. The results suggest that C/PGA PEMs provide a useful platform for modulating the micro-environment for better cell adhesion and antibiotic delivery, which hold great potential for surface modification and drug loading for biomimetic materials.
\end{abstract}

Keywords: polyelectrolyte multilayers; titanium alloy; 316L stainless steel; chitosan; poly- $\gamma$-glutamic acid

\section{Introduction}

For biomaterials functioning as main structure of implants, the mechanical and physical properties are the primary consideration for the selection of materials; however, the hard and bio-incompatible surface can inhibit the normal growth of human tissues [1], such as cell attachment and inhomogeneous growth due to surface roughness [2], and hence the usability of most structural materials is limited, especially for long-term clinical applications. Therefore, surface modification to create a biocompatible environment suitable for cell growth is the key for future development of these implants.

One of the most common practices is depositing polyelectrolyte multilayers to enhance cell viability and adhesion, while maintaining the structural strength of the overall implants. The concept of polyelectrolyte multilayers (PEMs) was first introduced in 1966 [3], and it has been widely applied in some of the most recent developments on photochemical disruption [4], drug loading control [5] and antibacterial applications [6-10]. PEMs are formed by alternating layer-by-layer deposition of 
positively and negatively charged polyelectrolytes in a self-assemble manner on solid supports [11]. PEMs demonstrate great benefits in biomedical engineering including low-temperature fabrication, adjustable mechanical strength, excellent biocompatibility, and controllable drug loading and release. PEMs have been applied as functional coatings for endowing titanium implants with bactericidal property and better anchorage in bone [12,13]. In addition, PEMs can provide an in vitro biomimetic environment, which can be used in tissue engineering.

We utilized positively charged chitosan and negatively charged poly- $\gamma$-glutamic acid for the proposed chitosan/poly-glutamic acid (C/PGA) polyelectrolyte multilayer coating. Chitosan (CTS) is a promising natural polymer for various biomedical applications. It is extracted by de-acetylation of chitin, which is the main element in the exoskeleton of crustaceans. Due to its solubility in acidic solution by forming the $-\mathrm{NH}_{2}$ functional group, chitosan is a natural cationic polymer [14], and it has been widely used as antibacterial agents, wound dressing materials, drug carriers and in plastic surgery due to its excellent biodegradable ability $[15,16]$. Another polymer chosen for this study is poly- $\gamma$-glutamic acid (PGA), which is a natural, non-toxic, highly biocompatible and edible polymer; it is often used in the food and medicine industries $[17,18]$. Currently, PGA is mostly synthesized by microbial fermentation from biomass. With the presence of the $\mathrm{COO}^{-}$functional group, good biodegradability and excellent water absorption ability, PGA attracts attention for drug delivery [19-21], and is selected as the negatively charged polyelectrolyte for the PEMs coating.

In the present study, we prepared C/PGA PEMs using simple spin coating technique to deposit the coating loaded with tetracycline on two different clinically used metals, Ti6Al4V titanium alloy (Ti6Al4V) and 316L stainless steel (316LSS). The microstructure, mechanical properties, biocompatibility and drug release rate were investigated by nanoindentation, scanning electron microscopy (SEM) and contact angle measurement. The biocompatibility of the PEMs was evaluated by the cell viability and attachment of MG63 cells while the antibacterial property of loaded tetracycline was studied by the growth inhibition of Escherichia coli (E. coli).

\section{Experimental Section}

\subsection{Materials}

CTS $(\mathrm{Mw}=300 \mathrm{kDa})$ and PGA $(\mathrm{Mw}=3840 \mathrm{kDa})$ were purchased from Charming \& Beauty Co., Ltd (Taipei, Taiwan) and Vedan Vietnam Enterprise Co., Ltd (Dong Nai Province, Vietnam). Dulbecco's Modified Eagle's Medium (DMEM), phosphate buffered saline (PBS) and fetal bovine serum (FBS) were provided by Gibco (Thermo Fisher Scientific Corporation, Waltham, MA, USA). Tetracycline, 3-(4,5-dimethylthiazol-2-yl)-2,5-diphenyl tetrazolium bromide (MTT), dimethyl sulfoxide (DMSO) and Hoechst 33258 were obtained from Sigma-Aldrich (Billerica, MA, USA). MG63 cell line (human osteosarcoma, ATCC CRL-1427) and E. coli (ATCC 25922) were purchased from American Type Culture Collection (Manassas, VA, USA). All other chemicals were obtained from Showa (Saitama, Japan) or Sigma-Aldrich if not specified.

\subsection{Preparing of Coating}

In this research, a layer-by-layer spin coating technique was used to stack the positively charged CTS and negatively charged PGA on two different alloy substrates, 316LSS and Ti6Al4V, as illustrated in Figure 1. Prior to deposition, 316LSS and Ti6Al4V substrates of diameter of $1.5 \mathrm{~cm}$ were grinded with No. 80 sand paper and ultrasonic bathed with acetone, ethanol and deionized water each for $10 \mathrm{~min}$ and then dried at $37^{\circ} \mathrm{C}$. CTS and PGA solutions were prepared by mixing $0.25 \mathrm{mg}$ powder each with $50 \mathrm{~mL}$ deionized water separately. An additional $100 \mu \mathrm{L}$ acetic acid was added in the chitosan solution to adjust the $\mathrm{pH}$. The substrate was first dipped in chitosan solution to form an overall protective layer, and then $150 \mu \mathrm{L}$ of the PGA solution was spin coated at $4000 \mathrm{rpm}$ onto the substrate, and the same process went on for the second chitosan layer. The substrate was dried at $37^{\circ} \mathrm{C}$ between each layer of coating. The same process was continued to prepare designated chitosan/poly-glutamic acid 
polyelectrolyte (C/PGA) multilayers of 10, 20 and 30. Finally, the coated specimen surface was cleaned with deionized water and dried again at $37^{\circ} \mathrm{C}$.
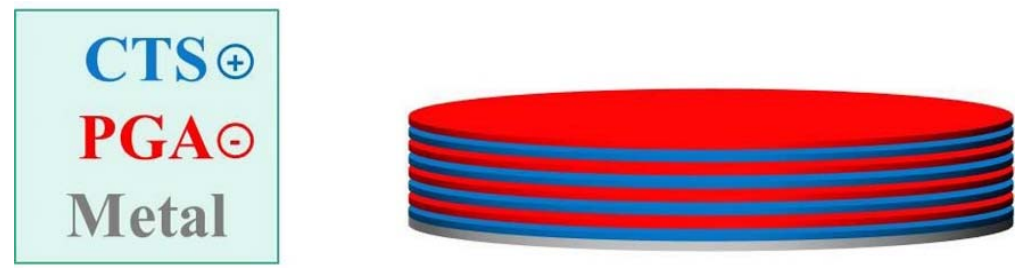

Figure 1. The illustration of chitosan/poly-glutamic acid polyelectrolyte (C/PGA) multilayers.

\subsection{Characterization}

The surface morphology, wettability and mechanical property of the coated substrates were characterized. The surface morphology and film thickness of the coating was observed using SEM (S-3000H, HITACHI, Tokyo, Japan). For the measurement of film thickness, half of the substrate was covered by adhesive tape prior to C/PGA coating which the thickness can be measured by a peel-off method. The specimens were sputter-coated with a 100 Å layer of gold-palladium for SEM imaging. The contact angle of the surface was measured using the sessile drop method with a contact angle analyzer (FTA1000, First Ten Angstroms, Cambridge, UK). Nano-indentation was performed with a nano-indenter (TI 700 Ubi, Hysitron, Minneapolis, MN, USA) equipped with a Berkovich pyramid tip to find out the hardness and reduced Young's modulus of the coating.

\subsection{Biocompatibility}

The biocompatibility of the coating was assessed by the cell viability and cell morphology of MG63 cells. MG63 cells were cultured in DMEM supplemented with 10\% FBS and 1\% penicillinstreptomycin-neomycin in a $37{ }^{\circ} \mathrm{C}$ incubator with $95 \% \mathrm{CO}_{2}$. C/PGA coated specimens were placed in the wells of a 24-well plate and seeded with $5 \times 10^{4}$ cells/well. After two days, the cell viability was examined using MTT assay by measuring the metabolic activity of the cells. The absorbance of the assay was read at $570 \mathrm{~nm}$ against a reference value of $630 \mathrm{~nm}$ as expressed as the optical density (OD) using a micro plate reader (Tecan, Sunrise remote F039300, Männedorf, Switzerland). The ODs measured were all normalized to the OD of cells cultured on uncoated metal surface. After one and three days in culture, MG63 cells were stained with Hoechst 33258 for observation of the cell morphology on the specimens. The specimens were then imaged with a fluorescence microscope (Nikon, Tokyo, Japan).

\subsection{Antibiotic Release and Antibacterial Activity}

Lastly, the drug release performance of C/PGA multilayers was evaluated by tetracycline release and zone of inhibition. Specimens were soaked in a saturated tetracycline solution for 12 hours and cleaned with deionized water. Then, the specimens were soaked with $5 \mathrm{~mL}$ PBS for 12 hours with sample collection $(100 \mu \mathrm{L})$ at every hour. The amount of tetracycline released was measured utilizing fluorescence micro plate reader (Varioskan Flash, Thermo Fisher Scientific Corporation, Waltham, MA, USA) with excitation wavelength at $360 \mathrm{~nm}$ and emission wavelength at $560 \mathrm{~nm}$. Inhibitory effect of tetracycline loaded coating was examined with E. coli. E. coli was cultured in Luria-Bertani (LB) broth and agar (Sigma-Aldrich, Billerica, MA, USA). LB agar was spread with $1 \times 10^{4} \mathrm{E}$. coli using a glass L-shaped cell spreader. Tetracycline loaded specimens were placed on the agar, and the agar plate was incubated at $37^{\circ} \mathrm{C}$ for 12 hours. Pictures were taken for measuring size of the zone of inhibition. 


\subsection{Statistical Analysis}

All numeric data were expressed as mean \pm SD with the sample size $n=5$. Unpaired-sample $t$-test was used to assess statistical significance of MTT results and zone of inhibition with $p<0.05$. The results of each group were compared to the corresponding bare metal substrate for analysis. Statistical analysis was performed using SPSS 17.0 software (IBM, Armonk, NY, USA).

\section{Results and Discussion}

\subsection{C/PGA Coating Morphology and Thickness}

The surface morphologies of the multilayers partially covered specimens are shown in Figure 2, and the corresponding cross sectional images are depicted in Figure 3. The C/PGA coatings with different layers of coating on Ti6Al4V and 316LSS are labelled as Ti-nL and S-nL, which n equals to 10, 20 or 30 layers. A smooth and uniform multilayered surface compared to the rough metal substrate can be observed in Figure 2, which is simply due to the liquid spin coating that fills the coarse substrate surface. The total thicknesses of 10, 20 and 30 layers of C/PGA PEMs on Ti6Al4V and 316LSS are about $2.3,4.7-4.9$ and $6.5-6.9 \mu \mathrm{m}$ with the exact values shown in Figure 3. As the coating layer increased, the film thickness increased linearly despite of the substrate material, and each layer of C/PGA coating was found to be around $230 \mathrm{~nm}$. The result suggests that this C/PGA coating by simple spin coating technique can be used to easily modify the biomedical metal surfaces in a tunable manner. The similar degree of hydrophilicity (will be discussed later) can also be the reason for the same film thickness of these two metal substrates.
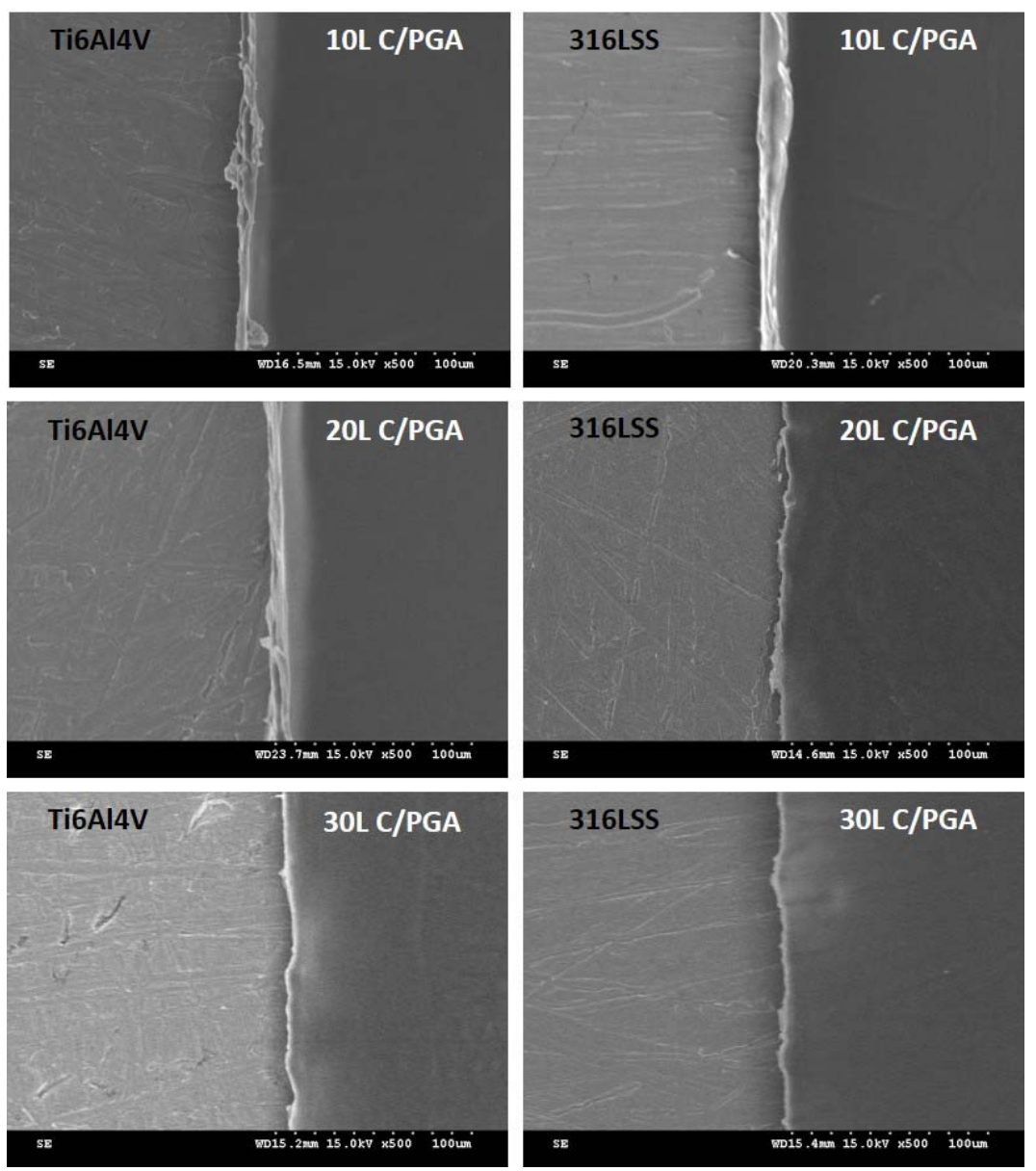

Figure 2. The SEM images of different layers of C/PGA on Ti6Al4V and 316LSS: The images are labelled as nL C/PGA with $\mathrm{n}$ indicating 10, 20 and 30 layers of C/PGA PEMs coating. 

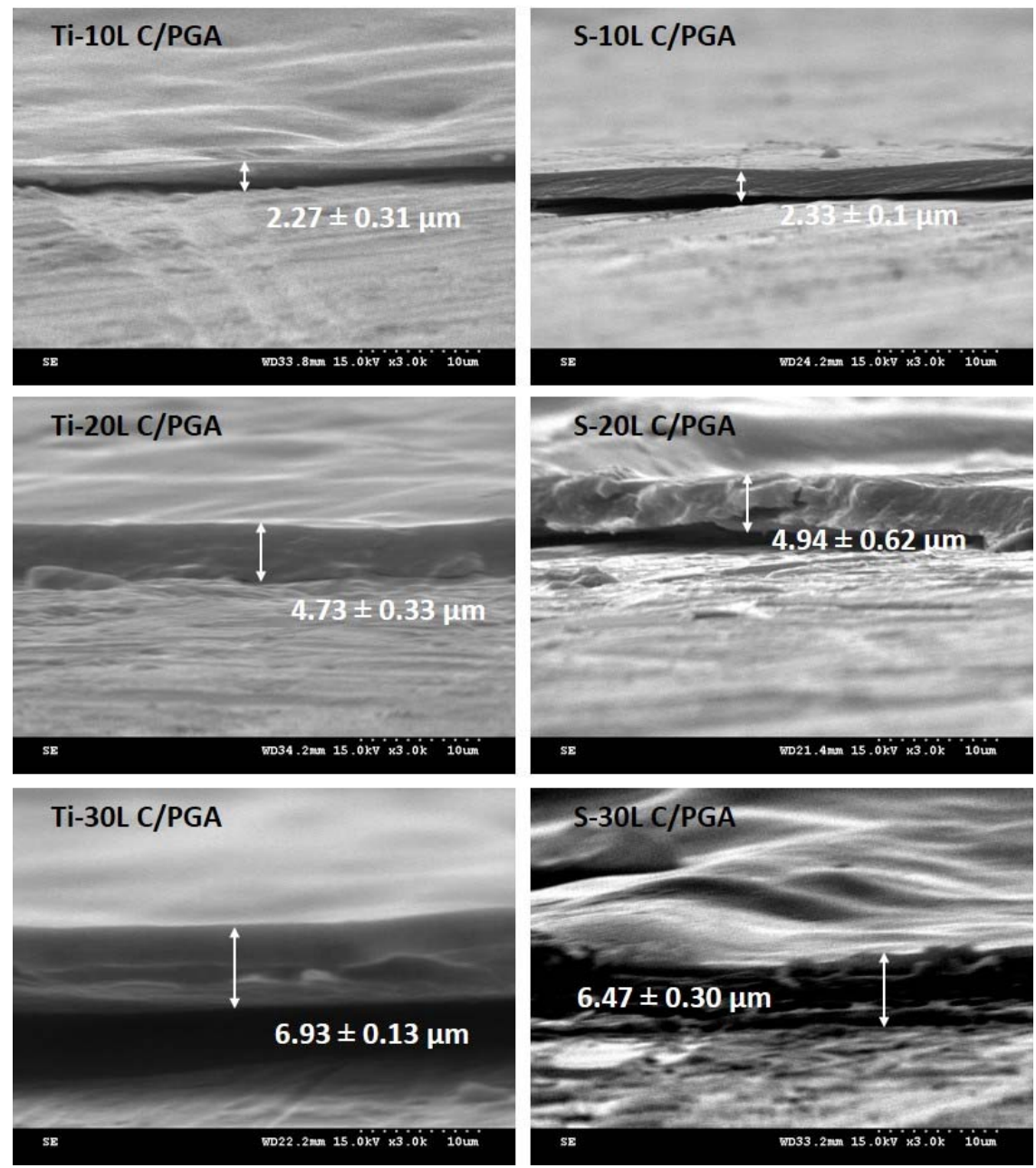

Figure 3. SEM cross-sectional images of different layers of C/PGA on Ti6Al4V and 316LSS substrates with the thickness of the coating measured. No statistical difference was found between Ti6Al4V and 316LSS substrates of the same number of layers.

\subsection{Water Contact Angle Measurement}

The water contact angle is used to measure the hydrophilicity of the C/PGA coating. Since the spin coating was performed with aqueous solutions, so similar contact angles of the coatings on Ti6Al4V and 316LSS, corresponding to $77.7^{\circ}$ and $75.7^{\circ}$ (Figure 4), were found. Due to the hydrophobic nature of chitosan [22], an obvious increase of contact angle to water could be found after adding the first chitosan layer onto the substrate. Further increased the C/PGA PEMs to a number of 10, two different finishing top coatings were made to study the wettability of the coating using CTS or PGA as the outer layer. The specimens coated with either CTS or PGA as the finishing layer are named as Ti-10L-CTS and S-10L-CTS, and those with PGA as the final coating are called Ti-10L-PGA and S-10L-PGA. As shown in Figure 4, the contact angles decreased dramatically to $45^{\circ}$ regardless of the final outer coatings indicating that chitosan becomes more hydrophilic when adding PGA multilayers. As the coating further increased to 20 and 30 layers, a saturated contact angle of $24^{\circ}$ was found. Stacking of positively and negatively charged polymers could lead to a rearrangement of local charge density and functional groups, and thus creating a hydrophilic surface, which could be beneficial for the adhesion of transmembrane glycoproteins and cell attachment [23]. 

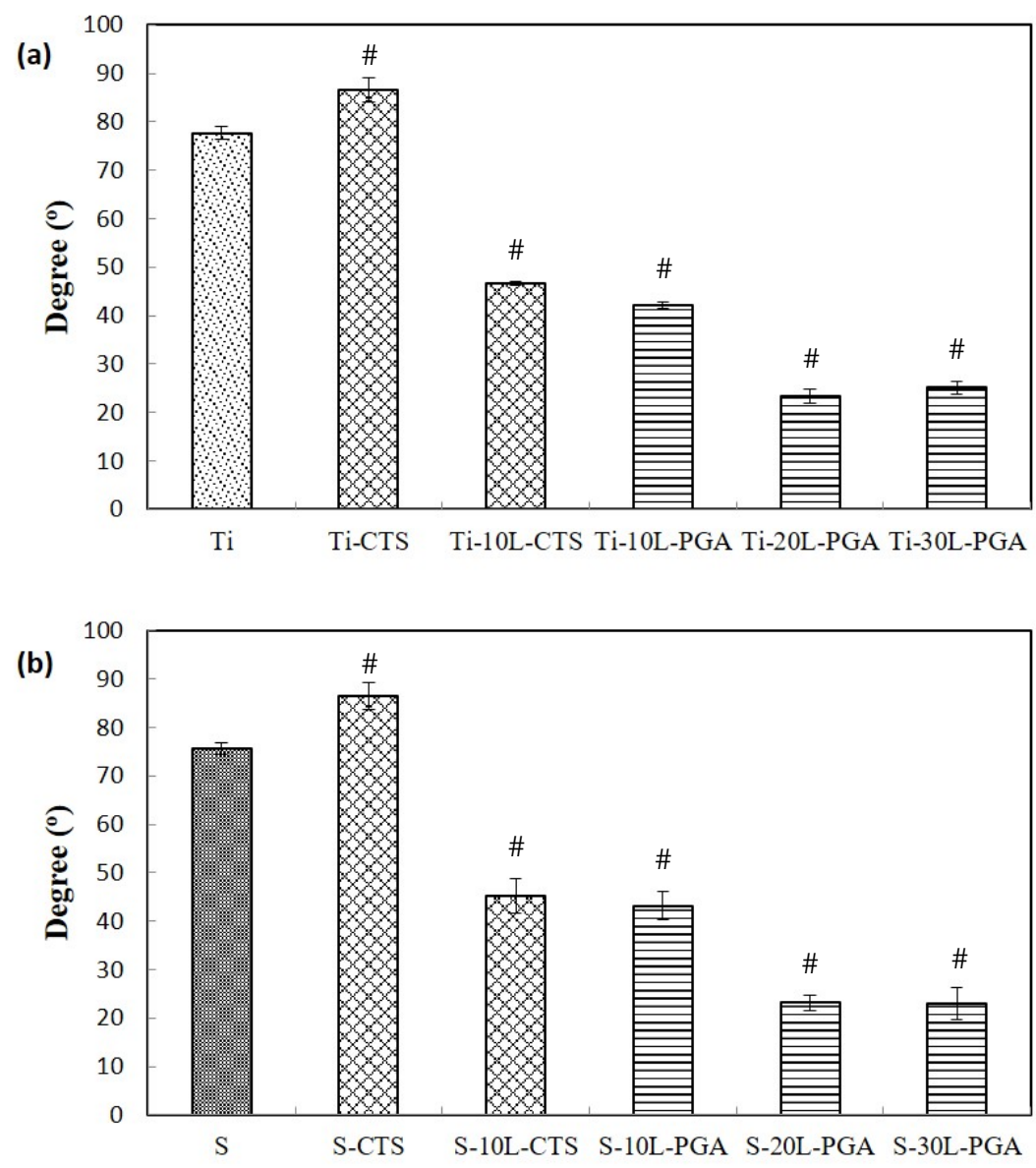

Figure 4. Contact angles with respect to water of (a) Ti6Al4V substrate with different layers of C/PGA multilayer coatings, and (b) 316LSS substrate with different layers of PEMs coating. \#: $p<0.01$ compared to bare Ti6Al4V or 316LSS substrate.

\subsection{Mechanical Properties}

The hardness and reduced Young's modulus were measured using nano-indentation with the results listed in Table 1. By adding only 10 layers of multilayers, the hardness and reduced Young's modulus dropped to less than $1 / 10$ or even more of the values of bare substrate materials, especially for 316LSS, suggesting a clear surface modification by C/PGA coating. Since the measurement utilized a constant indentation load of $100 \mu \mathrm{N}$, the hardness and reduced Young's modulus were assessed from the near-surface material; hence, the better mechanical properties were found in the groups with chitosan as the outer layer. Ti-10L-CTS and S-10L-CTS with chitosan as the outer layers are shown with higher reduced Young's modulus ranged from 4.5 to $4.7 \mathrm{GPa}$ as compared with those PGA covered specimens of 3.0-3.9 GPa. For cells to attach onto implant biomaterials, having the hardness of the materials comparable to the extracellular matrix is an important factor, in which this is totally contrary to the requirement of hard and tough metal-based structural implants [24]. With the PEMs coating, the hardness of the material substrates could be greatly reduced to about $1 / 3$ of that of bone tissue ( 0.6 to $0.8 \mathrm{GPa})[25,26]$, therefore, this C/PGA coating can be a simple and practical approach to modify the surface mechanical properties of biometals. 
Table 1. Hardness and reduced Young's modulus of the substrates with and without PEMs coating. The specimens are labeled according to the order of substrate material, number of layers and the outer coating material.

\begin{tabular}{ccc}
\hline Group & Reduced Young's Modulus $(G P a)$ & Hardness $(\mathbf{M P a})$ \\
\hline Ti6Al4V (Ti) & $39.5 \pm 5.5$ & $4610 \pm 39$ \\
Ti-10L-CTS & $4.5 \pm 0.2$ & $234 \pm 4$ \\
Ti-10L-PGA & $3.6 \pm 0.2$ & $203 \pm 4$ \\
Ti-20L-PGA & $3.9 \pm 0.2$ & $201 \pm 4$ \\
Ti-30L-PGA & $3.9 \pm 0.2$ & $224 \pm 4$ \\
316LSS (S) & $53.9 \pm 3.6$ & $3113 \pm 54$ \\
S-10L-CTS & $4.7 \pm 0.2$ & $244 \pm 5$ \\
S-10L-PGA & $3.0 \pm 0.1$ & $215 \pm 3$ \\
S-20L-PGA & $3.4 \pm 0.1$ & $244 \pm 3$ \\
S-30L-PGA & $3.9 \pm 0.1$ & $238 \pm 4$ \\
\hline
\end{tabular}

\subsection{Biocompatibility}

The biocompatibility of C/PGA PEMs coating was examined by MG63 cell viability and cell morphology seeded directly on the material surface. The cell viability of MG63 cultured on different $\mathrm{C} / \mathrm{PGA}$ groups is reported with OD values from MTT assay while the OD values were normalized to the OD of the corresponding metal substrate without coating as shown in Figure 5a,b. A slight reduction of cell viability without significant difference is observed with Ti-10L-CTS and S-10L-CTS using chitosan as the outer coating layer. The result of chitosan coating on titanium based surface not promoting cell proliferation is in good agreement with other studies [27-29]. While both Ti6Al4V and 316LSS are widely used orthopedic implants known with good biocompatibility, it is not a surprise that these surfaces favor cell growth in this study. On the other hand, by coating PGA as the outer layer, the increase of cell viability of all groups demonstrates that C/PGA PEMs coated substrates actually promote MG63 cell proliferation. The highest cell proliferation was founded in 30 layers PGA coating groups with the cell viability enhanced with $40 \%-58 \%$ with statistical difference. PGA, which carries a negatively charged carboxyl group, has been suggested to improve the hydrophilicity, biocompatibility and degradation rate of chitosan in the form as a composite; therefore, the presence of PGA can promote cell attachment and proliferation [30-33].

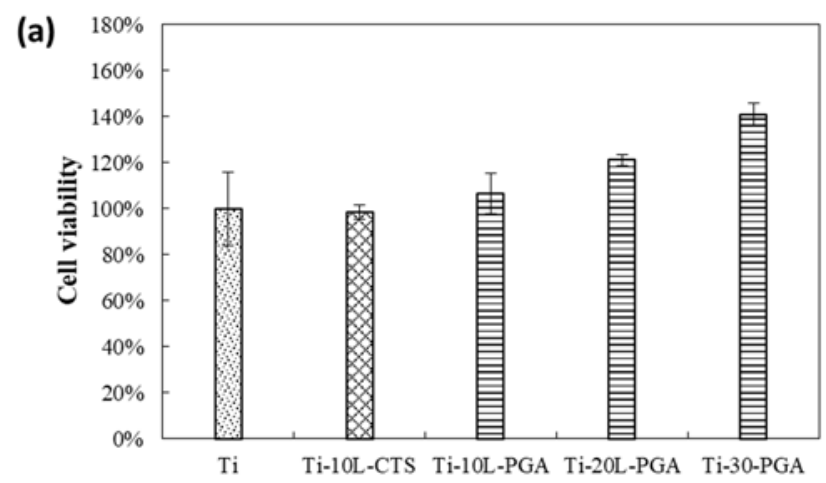

Figure 5. Cont. 


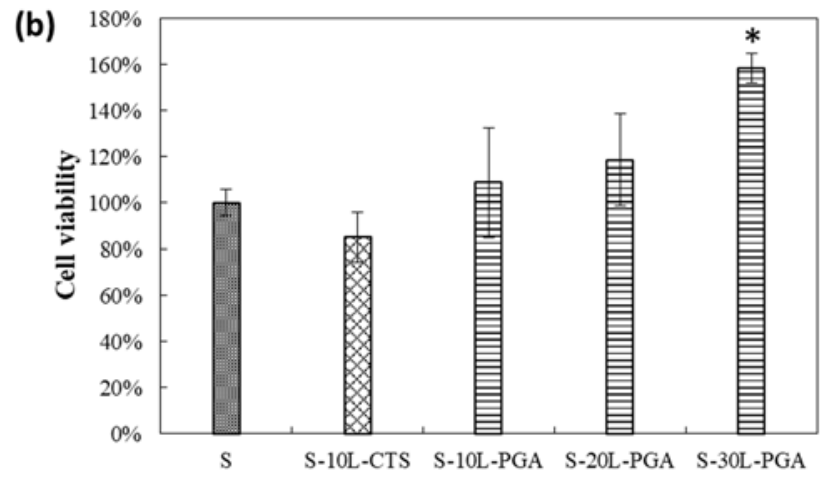

Figure 5. The cell viability of MG63 seeded on (a) Ti6Al4V substrate and (b) 316LSS substrate with different layers of PEMs coatings were measured using MTT assay. The bare metal substrate was used as the control. *: $p<0.05$ compared to bare Ti6Al4V or 316LSS substrate.

A more direct method to observe the cell adhesion and affinity on a material is to observe cell attachment and morphology using fluorescence microscopy. MG63 cells seeded on different layers of C/PGA PEMs coating on Ti6Al4V and 316LSS substrates for one and three days are depicted in Figures 6 and 7. At the first day (Figure 6), both bare metal substrates and PEMs coated specimens show minimal cell adhesion. Only a few MG63 cells were found on the specimens with PGA as the outer layers. After three days of culturing, both bare metal substrates and PEMs coated specimens demonstrate different degrees of cell attachment as shown in Figure 7. More MG63 cells were observed on bare 316LSS surface as compared to bare Ti6Al4V. The PEMs coated specimens with chitosan as the outer layer show similar affinity of cell adhesion as bare metal substrates, which is in coherence with the cell viability result. Polymeric materials are known to hinder fluorescence imaging due to either autofluorescence or light scattering property of polymers [34]; thus, it is not possible to clearly identify the cells on PEMs coated specimens with PGA as the finishing layer in this experiment.

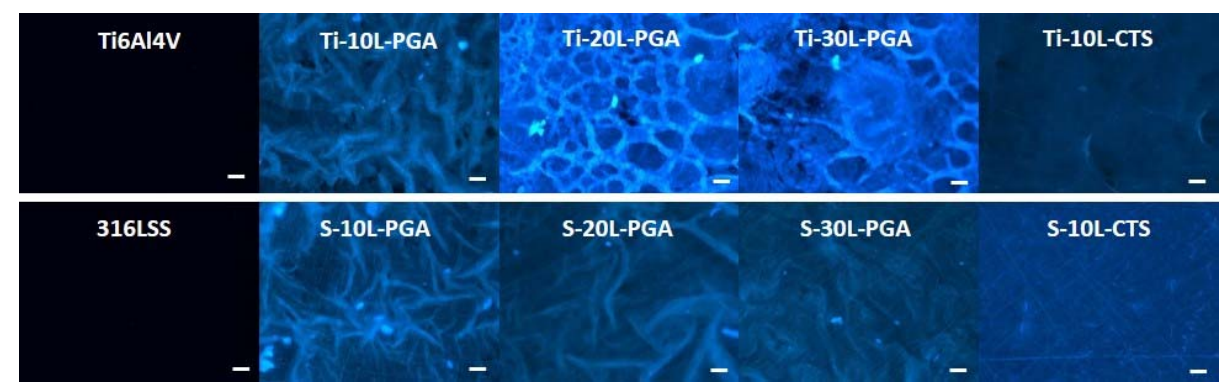

Figure 6. Fluorescence microscopy images of the MG63 cells seeded on different layers of C/PGA on Ti6Al4V and 316LSS substrates for one day and stained with Hoechst 33258. The scale bars are labeled in white with the length of $100 \mu \mathrm{m}$.
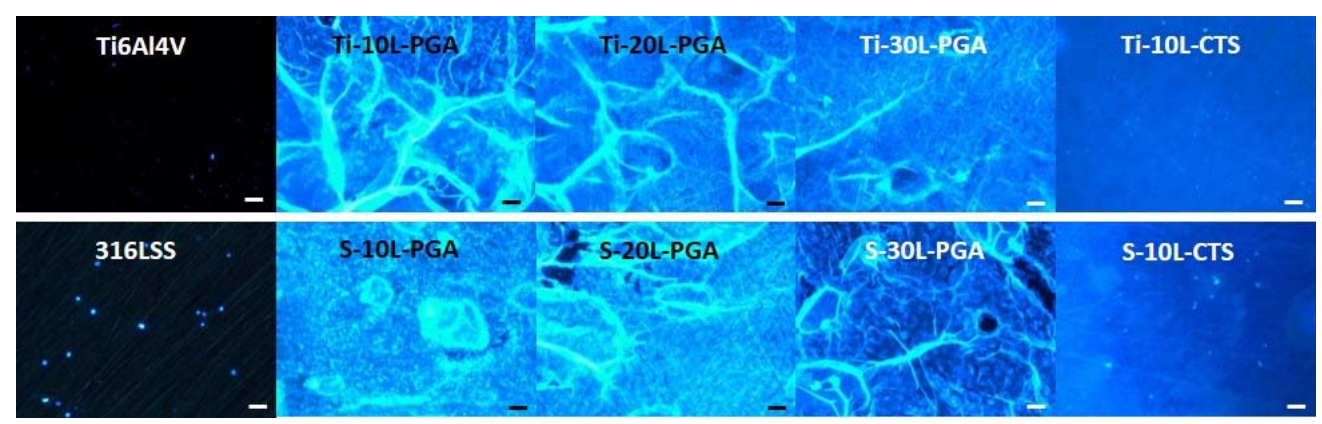

Figure 7. Fluorescence microscopy images of the MG63 cells seeded on different layers of C/PGA on Ti6Al4V and 316LSS substrates for three days and stained with Hoechst 33258. The scale bars are labeled in white or black with the length of $100 \mu \mathrm{m}$. 


\subsection{Tetracycline Release and Antibacterial Activity}

Lastly, the drug release performance of C/PGA PEMs was carried out to investigate the tetracycline release rate and the effect of tetracycline released on $E$. coli inhibition. Release of tetracycline from bare metal substrates and substrates with different PEMs coating were depicted in Figure 8. In general, bare metal substrates and substrates coated with C/PGA PEMs with CTS as the outer layer demonstrate equally minimal release of tetracycline, which suggests that both metal surfaces and CTS are not suitable for drug loading. However, by modifying Ti6Al4V and 316LSS surfaces with PEMs coating with PGA as the outer layers, the drug loading ability of these metals can be greatly improved. Moreover, the release of tetracycline increased with the layers of PEMs for PGA groups. However, the release of tetracycline reaches a plateau within the first three hours, which suggests that this PEMs coating needs to be further investigated for longer drug release usage.
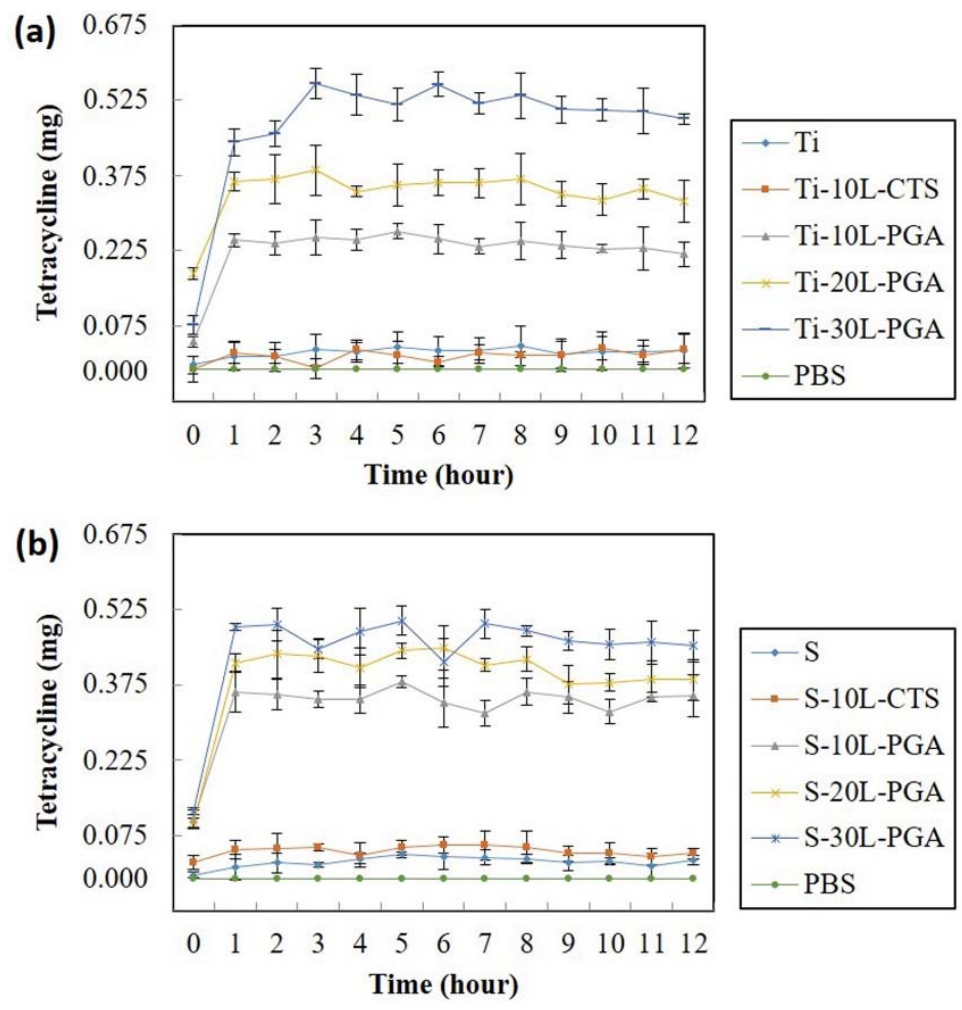

Figure 8. The release of tetracycline from (a) Ti6Al4V and (b) 316LSS substrates with different layers of PEMs coating.

The antibacterial effect of this PEMs coating is demonstrated by the inhibition of $E$. coli growth as shown in Figure 9. Larger inhibition zones can also be observed as the layers of PEMs increased, which are due to the fact that more drug can be stored within thicker films, especially in PGA layers, as confirmed by the tetracycline release measurement. For both CTS and PGA as the outer layer of 10 layers of PEMs coating, the antibacterial properties of both metal-10L-CTS and metal-10L-PGA are much better than bare metal substrates. However, metal-10L-PGA performed better as compared with metal-10L-CTS, which can be explained by the tetracycline result in the previous section. Tetracycline could be successfully loaded and released from PEMs coating with PGA as the outer layer while very little tetracycline was released from the CTS outer layer PEMs coating; thus, the antibacterial ability of the coating should be primarily contributed by the well-documented intrinsic antibacterial nature of chitosan [35]. The initial design of the antimicrobial ability of this current PEMs coating was primarily contributed by the tetracycline loaded. However, other than release of antimicrobial agents, the versatility of PEMs technology can also limit microbial colonization by functionalizing the coatings 
as adhesion-resistance and contact killing property (Lichter 09; Seon 15). PEMs terminated by CTS have been reported to possess antibacterial properties by contact killing (Bratskaya 07). Herewith, further studies with antimicrobial PEMs coating should also consider the interplay between the choice of polyelectrolytes and antibiotic agents.
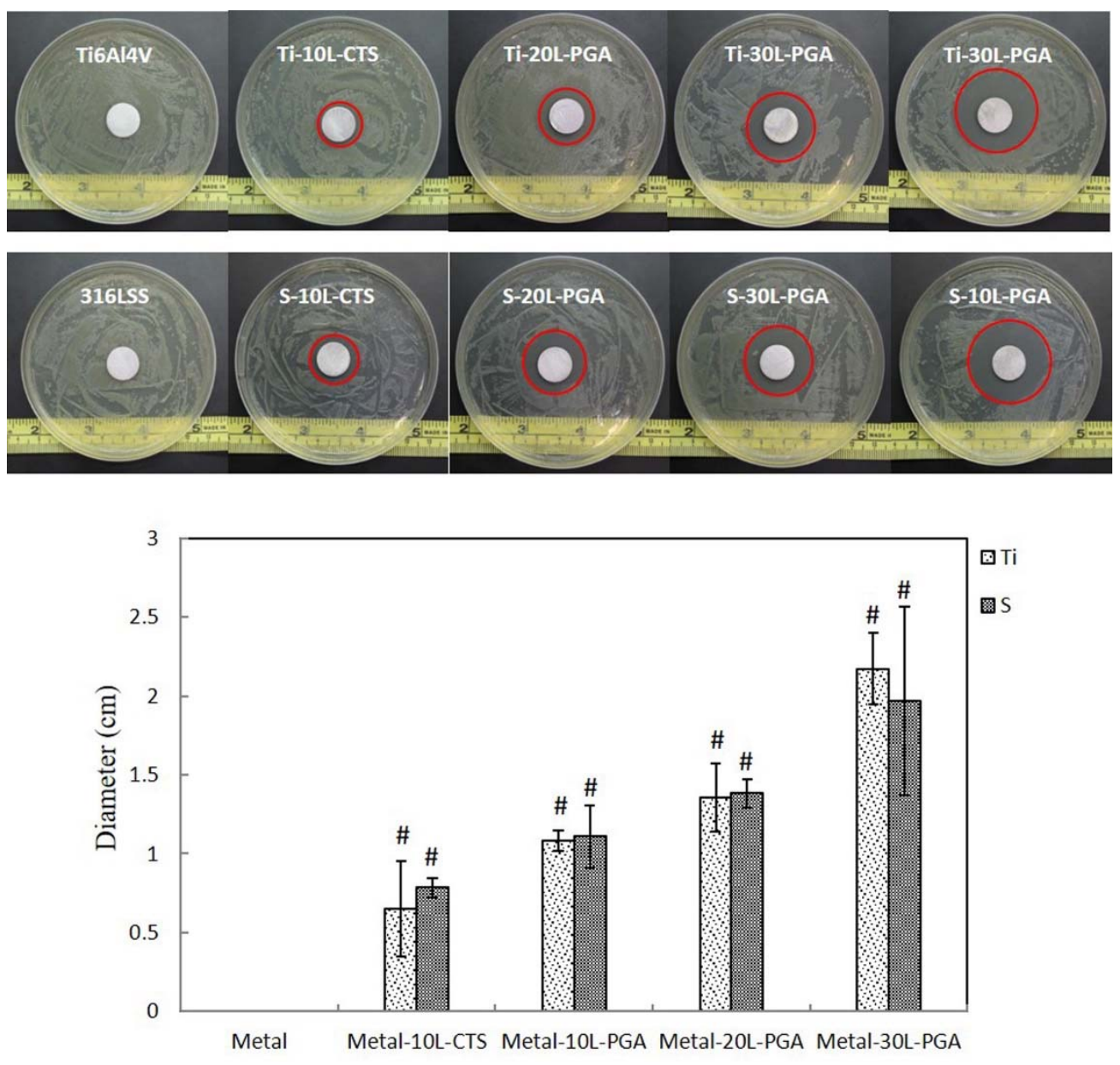

Figure 9. Growth inhibition of E. coli by tetracycline released from Ti6Al4V and 316LSS substrates without and with PEMs coating. The diameter of the zone of inhibition was measured and presented here. \#: $p<0.01$ compared to bare Ti6Al4V or 316LSS substrate.

\section{Conclusion}

In this research, the chitosan/poly-glutamic acid polyelectrolytes multilayer coating was successfully prepared using simple spin coating technique deposited on two different clinically used metals, Ti6Al4V and 316LSS. The surface of the metal substrate was modified with the intention to mimic extracellular matrix to support cell growth. The mechanical properties and hydrophilicity of the metal substrates were evidently changed with PEMs coating. Moreover, an excellent biocompatibility with over $40 \%$ enhancement of cell growth was achieved with 30 layers of C/PGA with PGA as the outer layers. Last but not least, this C/PGA PEMs coating was demonstrated to carry tetracycline with antibacterial ability. To sum up, we demonstrate this C/PGA PEMs coating with PGA outer layer having great potential as a biomimicry material for surface modification and drug delivery.

Acknowledgments: The authors are grateful for the financial support of this research by the Ministry of Science and Technology of the Republic of China, under Grant No. MOST 104-2221-E-027-061 and MOST 106-2221-E-027-034; and part from the National Taipei University of Technology-Mackay Memorial Hospital Joint Research, under Grant No. MMH-TT-10401, NTUT-MMH-105-02 and NTUT-MMH-106-05. The first two authors contributed equally to this work. 
Author Contributions: Wai-Ching Liu wrote the article and analyzed part of the data; Huey-Yuan Wang supported and analyzed part of the data; An-Ni Wang wrote the article and analyzed part of the data; Chih-Hsien Tseng performed the experiments, measurements, and data analyses; Hsuan-Liang Liu supported and analyzed part of the data; Ren-Jei Chung organized and supervised this work.

Conflicts of Interest: The authors have declared that there is no conflict of interest.

\section{References}

1. Guduru, D.; Niepel, M.; Vogel, J.; Groth, T. Nanostructured material surfaces-preparation, effect on cellular behavior, and potential biomedical applications: A review. Int. J. Artif. Organs 2011, 34, 963-985. [CrossRef] [PubMed]

2. Costa, R.; Mano, J. Polyelectrolyte multilayered assemblies in biomedical technologies. Chem. Soc. Rev. 2014, 43, 3453-3479. [CrossRef] [PubMed]

3. Iler, R. Multilayers of colloidal particles. J. Colloid Interface Sci. 1966, 21, 569-594. [CrossRef]

4. Koylu, D.; Thapa, M.; Gumbley, P.; Thomas, S.W. Photochemical disruption of polyelectrolyte multilayers. Adv. Mater. 2012, 24, 1451-1454. [CrossRef] [PubMed]

5. Ding, C.; Xu, S.; Wang, J.; Liu, Y.; Chen, P.; Feng, S. Controlled loading and release of methylene blue in layer-by-layer assembled polyelectrolyte films. Mater. Sci Eng. C Mater. Biol. Appl. 2012, 32, 670-673. [CrossRef]

6. De Paiva, R.; de Moraes, M.; de Godoi, F.; Beppu, M. Multilayer biopolymer membranes containing copper for antibacterial applications. J. Appl. Polym. Sci. 2012, 126, E17-E24. [CrossRef]

7. Hizal, F.; Zhuk, I.; Sukhishvili, S.; Busscher, H.; van der Mei, H.; Choi, C. Impact of 3D Hierarchical Nanostructures on the Antibacterial Efficacy of a Bacteria-Triggered Self-Defensive Antibiotic Coating. ACS Appl. Mater. Interfaces 2015, 7, 20304-20313. [CrossRef] [PubMed]

8. Monge, C.; Almodóvar, J.; Boudou, T.; Picart, C. Spatio-Temporal Control of LbL Films for Biomedical Applications: From 2D to 3D. Adv. Healthc. Mater. 2015, 4, 811-830. [CrossRef] [PubMed]

9. Herron, M.; Schurr, M.; Murphy, C.; McAnulty, J.; Czuprynski, C.; Abbott, N. Interfacial Stacks of Polymeric Nanofilms on Soft Biological Surfaces that Release Multiple Agents. ACS Appl. Mater. Interfaces 2016, 8, 26541-26551. [CrossRef] [PubMed]

10. Wei, T.; Zhan, W.; Cao, L.; Hu, C.; Qu, Y.; Yu, Q.; Chen, H. Multifunctional and Regenerable Antibacterial Surfaces Fabricated by a Universal Strategy. ACS Appl. Mater. Interfaces 2016, 8, 30048-30057. [CrossRef] [PubMed]

11. Decher, G. Fuzzy Nanoassemblies: Toward Layered Polymeric Multicomposites. Science 1997, 277, $1232-1237$. [CrossRef]

12. Moskowitz, J.; Blaisse, M.; Samuel, R.; Hsu, H.; Harris, M.; Martin, S.; Lee, J.; Spector, M.; Hammond, P. The effectiveness of the controlled release of gentamicin from polyelectrolyte multilayers in the treatment of Staphylococcus aureus infection in a rabbit bone model. Biomaterials 2010, 31, 6019-6030. [CrossRef] [PubMed]

13. Zankovych, S.; Diefenbeck, M.; Bossert, J.; Mückley, T.; Schrader, C.; Schmidt, J.; Schubert, H.; Bischoff, S.; Faucon, M.; Finger, U.; et al. The effect of polyelectrolyte multilayer coated titanium alloy surfaces on implant anchorage in rats. Acta Biomater. 2013, 9, 4926-4934. [CrossRef] [PubMed]

14. Rinaudo, M. Chitin and Chitosan: Properties and Applications. Prog. Polym. Sci. 2006, 31, $603-632$. [CrossRef]

15. Lin, Q.; Ren, K.; Ji, J. Hyaluronic acid and chitosan-DNA complex multilayered thin film as surface-mediated nonviral gene delivery system. Colloids Surf. B Biointerfaces 2009, 74, 298-303. [CrossRef] [PubMed]

16. Chen, X.; Liu, C.; Liu, C.; Meng, X.; Lee, C.; Park, H. Preparation and biocompatibility of chitosan microcarriers as biomaterial. Biochem. Eng. J. 2006, 27, 269-274. [CrossRef]

17. Sung, M.; Park, C.; Kim, C.; Poo, H.; Soda, K.; Ashiuchi, M. Natural and edible biopolymer poly-gamma-glutamic acid: Synthesis, production, and applications. Chem. Rec. 2005, 5, 352-366. [CrossRef] [PubMed]

18. Luo, Z.; Guo, Y.; Liu, J.; Qiu, H.; Zhao, M.; Zou, W.; Li, S. Microbial synthesis of poly- $\gamma$-glutamic acid: Current progress, challenges, and future perspectives. Biotechnol. Biofuels 2016, 9, 134. [CrossRef] [PubMed] 
19. Ho, G.; Ho, T.; Hsieh, K.; Su, Y.; Lin, P.; Yang, J.; Yang, K.; Yang, S. $\gamma$-Polyglutamic Acid Produced by Bacillus Subtilis (Natto): Structural Characteristics, Chemical Properties and Biological Functionalities. J. Chin. Chem. Soc. 2006, 53, 1363-1384. [CrossRef]

20. Paul, N.; Paul, A.; Steitz, R.; Kreuzer, M.; Lux-Steiner, M. Selective self assembly of glutamate molecules on polyelectrolyte multilayers. J. Phys. Chem. B 2012, 116, 4492-4499. [CrossRef] [PubMed]

21. Ogunleye, A.; Bhat, A.; Irorere, V.; Hill, D.; Williams, C.; Radecka, I. Poly- $\gamma$-glutamic acid: Production, properties and applications. Microbiology 2015, 161, 1-17. [CrossRef] [PubMed]

22. Philippova, O.; Korchagina, E. Chitosan and its hydrophobic derivatives: Preparation and aggregation in dilute aqueous solutions. Polym. Sci. Ser. A 2012, 54, 552-572. [CrossRef]

23. Kasemo, B. Biological surface science. Surf. Sci. 2002, 500, 656-677. [CrossRef]

24. Discher, D.; Janmey, P.; Wang, Y. Tissue cells feel and respond to the stiffness of their substrate. Science 2002, 310, 1139-1143. [CrossRef] [PubMed]

25. Zysset, P.; Guo, X.; Hoffler, C.; Moore, K.; Goldstein, S. Elastic modulus and hardness of cortical and trabecular bone lamellae measured by nanoindentation in the human femur. J. Biomech. 1999, 32, 1005-1012. [CrossRef]

26. Mathieu, V.; Vayron, R.; Richard, G.; Lambert, G.; Naili, S.; Meningaud, J.; Haiat, G. Biomechanical determinants of the stability of dental implants: influence of the bone-implant interface properties. J. Biomech. 2014, 47, 3-13. [CrossRef] [PubMed]

27. Yuan, Y.; Chesnutt, B.; Wright, L.; Haggard, W.; Bumgardner, J. Mechanical property, degradation rate, and bone cell growth of chitosan coated titanium influenced by degree of deacetylation of chitosan. J. Biomed. Mater. Res. B Appl. Biomater. 2008, 86, 245-252. [CrossRef] [PubMed]

28. Renoud, P.; Toury, B.; Benayoun, S.; Attik, G.; Grosgogeat, B. Functionalization of titanium with chitosan via silanation: Evaluation of biological and mechanical performances. PLoS ONE 2012, 7, e39367. [CrossRef] [PubMed]

29. Fang, K.; Song, W.; Wang, L.; Jia, S.; Wei, H.; Ren, S.; Xu, X.; Song, Y. Immobilization of chitosan film containing semaphorin $3 \mathrm{~A}$ onto a microarc oxidized titanium implant surface via silane reaction to improve MG63 osteogenic differentiation. Int. J. Nanomed. 2014, 9, 4649-4657.

30. Yan, S.; Zhang, K.; Liu, Z.; Zhang, X.; Gan, L.; Cao, B.; Chen, X.; Cui, L.; Yin, J. Fabrication of poly(L-glutamic acid)/chitosan polyelectrolyte complex porous scaffolds for tissue engineering. J. Mater. Chem. B 2013, 1, 1541-1551. [CrossRef]

31. Song, Z.; Yin, J.; Luo, K.; Zheng, Y.; Yang, Y.; Li, Q.; Yan, S.; Chen, X. Layer-by-layer buildup of poly(L-glutamic acid)/chitosan film for biologically active coating. Macromol. Biosci. 2009, 9, 268-278. [CrossRef] [PubMed]

32. Wu, H.; Yang, J.; Tsai, T.; Ji, D.; Chang, W.; Chen, C.; Lee, S. Development of a chitosan-polyglutamate based injectable polyelectrolyte complex scaffold. Carbohydr. Polym. 2011, 85, 318-324. [CrossRef]

33. Fang, J.; Zhang, Y.; Yan, S.; Liu, Z.; He, S.; Cui, L.; Yin, J. Poly(L-glutamic acid)/chitosan polyelectrolyte complex porous microspheres as cell microcarriers for cartilage regeneration. Acta Biomater. 2014, 10, $276-288$. [CrossRef] [PubMed]

34. Jaafar, I.; LeBlon, C.; Wei, M.; Ou-Yang, D.; Coulter, J.; Jedlicka, S. Improving fluorescence imaging of biological cells on biomedical polymers. Acta Biomater. 2011, 7, 1588-1598. [CrossRef] [PubMed]

35. Rabea, E.; Badawy, M.; Stevens, C.; Smagghe, G.; Steurbaut, W. Chitosan as antimicrobial agent: applications and mode of action. Biomacromolecules 2003, 4, 1457-1465. [CrossRef] [PubMed]

(C) 2017 by the authors. Licensee MDPI, Basel, Switzerland. This article is an open access article distributed under the terms and conditions of the Creative Commons Attribution (CC BY) license (http://creativecommons.org/licenses/by/4.0/). 